\title{
Salidroside Alleviates Lipid Metabolism Disorder and Inflammatory Response, and Promotes Hepatocyte Proliferation in Fatty Liver-Hemorrhagic Syndrome of Laying Hens
}

\section{Zhifu Cui}

Sichuan Agricultural University - Chengdu Campus

\section{Ningning Jin}

Sichuan Agricultural University - Chengdu Campus

Felix Kwame Amevor

Sichuan Agricultural University - Chengdu Campus

\section{Liang Li}

Sichuan Agricultural University - Chengdu Campus

\section{Gang Shu}

Sichuan Agricultural University - Chengdu Campus

\section{Xiaxia Du}

Sichuan Agricultural University - Chengdu Campus

\section{Xincheng Kang}

Sichuan Agricultural University - Chengdu Campus

\section{Dan Xu}

Sichuan Agricultural University - Chengdu Campus

\section{Zifan Ning}

Sichuan Agricultural University - Chengdu Campus

\section{Xun Deng}

Sichuan Agricultural University - Chengdu Campus

\section{Weizhen Song}

Sichuan Agricultural University - Chengdu Campus

Yaofu Tian

Sichuan Agricultural University - Chengdu Campus

\section{Qing Zhu}

Sichuan Agricultural University - Chengdu Campus

\section{Yan Wang}

Sichuan Agricultural University - Chengdu Campus

Diyan Li

Sichuan Agricultural University - Chengdu Campus 


\section{Yao Zhang}

Sichuan Agricultural University - Chengdu Campus

\section{Xiaoqi Wang}

Agriculture and Animal Husbandry Comprehensive Service Center of Razi County, Tibet Autonomous Region, P. R. China.

\section{Xue Han}

Guizhou Institute of Animal Husbandry and Veterinary Medicine, Guizhou Province, P. R. China.

\section{Jing Feng}

Institute of Animal Husbandry and Veterinary Medicine, College of Agriculture and Animal Husbandry,

Tibet Autonomous Region, P. R. China.

Xiaoling Zhao ( $\nabla$ zhaoxiaoling@sicau.edu.cn )

Sichuan Agricultural University - Chengdu Campus

\section{Research}

Keywords: Layer, Fatty liver hemorrhagic syndrome, Salidroside, Inflammatory response, Hepatocyte proliferation.

Posted Date: September 21st, 2021

DOI: https://doi.org/10.21203/rs.3.rs-885594/v1

License: (c) (1) This work is licensed under a Creative Commons Attribution 4.0 International License. Read Full License 


\section{Abstract}

Background: Fatty liver hemorrhagic syndrome (FLHS) is a chronic hepatic disease which occurs when there is a disorder in lipid metabolism. This disease is often observed in caged laying hens and characterized by a decrease in egg production and dramatic increase of mortality. Salidroside (SDS) is an herbal drug which has shown numerous pharmacological activities, such as protective effects on mitochondrial function, attenuates cell apoptosis and inflammation, and promotes strong antioxidant defense system. We aimed to determine the therapeutic effects of SDS on FLHS in laying hens and investigate the underlying mechanisms through which SDS operates these functions. We constructed oleic acid (OA)-induced fatty liver model in vitro and high-fat diet-induced FLHS of laying hens in vivo.

Results: Results indicated that SDS inhibited OA-induced lipid accumulation in chicken primary hepatocytes, increased hepatocyte activity, elevated the mRNA expression of proliferation related genes $P C N A, C D K 2$, and cyclinD1 and increased the protein levels of PCNA and CDK2, as well as decreased the cleavage levels of Caspase-9, Caspase- 8 , and Caspase- 3 and apoptosis in hepatocytes. Moreover, SDS promoted the phosphorylation levels of PDK1, AKT, and Gsk3- $\beta$, while inhabited the PI3K inhibitor. Additionally, we found that high-fat diet-induced FLHS of laying hens in vivo resulted in heavier body weight, liver weight, and abdominal fat weight, and severer steatosis in histology, compared with the control group (Con). However, SDS maintained lighter body weight, liver weight, and abdominal fat weight and alleviate hepatic steatosis in Model+SDS group. In addition, high-fat diet-induced FLHS (Model) of laying hens had higher total cholesterol (TC), triglyceride (TG), alanine transaminase (ALT), and aspartate aminotransferase (AST) levels in serum than Con group, while SDS maintained low TC, TG, ALT, and AST levels and high Superoxide dismutase (SOD) activity in Model+SDS group. Moreover, SDS decreased the mRNA expression abundances of PPARY, SCD, and FAS in liver, whereas increased those of PPARa and MTTP, and decreased the mRNA expression of TNF- $a, I L-1 \beta, I L-6$, and $I L-8$ in Model+SDS group.

Conclusions: Generally, SDS attenuated OA-induced ROS generation, inhibited lipid accumulation and hepatocyte apoptosis, and promoted hepatocyte proliferation by targeting the pathway PI3K/AKT/Gsk3- $\beta$ in OA-induced fatty liver model in vitro, and alleviated high-fat diet-induced hepatic steatosis, oxidative stress, and inflammatory response in laying hens in vivo.

\section{Introduction}

In poultry, liver has strong lipid synthesis ability but poor lipid storage capacity. Excessive storage of lipid substances in liver results in a series of pathological occurrences in aging chickens, in which the most representative disease is fatty liver-hemorrhagic syndrome (FLHS) of laying hens, which seriously decreases egg production [1-4]. Further deterioration of fatty liver may lead to steatohepatitis, liver fibrosis, and even cirrhosis [5], having sudden death of individuals in the layer flocks [6, 7].

Studies indicated that high fat diets led to fat transport obstruction in chicken liver, disrupted the dynamic balance of fat metabolism in hepatocytes, adipocytes, and bloods. Excessive deposition of lipids were 
mainly composed of neutral fat in hepatocytes [8], which is an important pathological feature of nonalcoholic fatty liver disease (NAFLD) [9-11]. Excessive lipid accumulation and estrogen secretion depressed the mitochondrial function in hepatocytes, and produced harmful reactive oxygen species (ROS) which damaged hepatocytes, and further reduced the activity of antioxidant enzymes in organisms by inhibiting gene transcription, thus causing damage to the antioxidant system of animals [12].

Plant extracts were reported to improve liver steatosis [13-15]. Rhodiola rosea L. radix (RRL) known as plateau ginseng is a rare medicinal herb that grows in cold high-altitude areas. In Ming Dynasty, China (five hundred years ago) RRL has used as a tonic to treat diseases, eliminate fatigue and resist cold. Modern pharmacological studies have confirmed that RRL extract has anti-aging, anti-tumor, antihypoxia, anti-fatigue, and anti-oxidation functions [16-21].

Salidroside (SDS) is the main pharmacological component of RRL. It can scavenge free radicals, decrease oxidative stress, enhance immunity, and prevent aging in animals [16-21]. Meanwhile, SDS has no genetic toxicity or mutagenesis [22]. Mao et al. induced the aging model with D-galactose in mice, and found that SDS could inhibit the formation of advanced glycation end products in vivo, which increased the production of lymphocyte mitosis and interleukin-2 (IL-2), and had an anti-aging effect [23]. Lu et al. studied the effects of SDS on the number of helper T cells and the delayed type hypersensitivity response in aged rats, and found that SDS stimulated the body's humoral and cellular immune responses [16]. Zhang found that SDS inhibited $\mathrm{H}_{2} \mathrm{O}_{2}$-induced cell viability loss, reduced cell apoptosis rate, inhibited mitochondrial membrane potential decline, and increased intracellular $\mathrm{Ca}^{2+}$ concentration, which can be used to treat and prevent neurodegenerative diseases [24]. Guan demonstrated SDS resisted lipopolysaccharide (LPS)-induced acute pneumonia in a mouse model [25]. Li reported that SDS prevented mouse mastitis induced by LPS, reduced the activity of myeloperoxidase and the concentrations of TNF- $a$, IL-1 $\beta$, and IL- 6 in breast muscle tissue, inhibited the inflammatory cell infiltration, and produced anti-inflammatory effects [26].

Additionally, SDS protected against LPS-induced liver failure in mice by its antioxidant activity and inhibited hypoxia-inducible factor-1a expression [27], protected against acetaminophen-induced hepatotoxicity by preventing or mitigating intracellular glutathione depletion and oxidative damage [28], and inhibited chronic hepatitis $C$ virus by inhibiting serine protease activity [29]. Recent studies indicated that SDS alleviated hypoxia-induced liver injury and inhibited cell apoptosis via the IRE1a/JNK pathway [30], alleviated carbon tetrachloride-induced liver damage in mice by activating mitochondria to resist oxidative stress [31], inhibited apoptosis and autophagy in a Concanavalin A-induced liver injury model [32], protected oxidative stress induced by diabetes mellitus, effectively reduced blood TC and TG levels [33], alleviated liver steatosis in type 2 diabetic mice [34], and reduced obesity and liver lipid deposition induced by a high-fat diet by inhibiting oxidative stress and activation of inflammatory bodies in the liver [35].

Generally, SDS protected liver and treated liver diseases in mammalian studies. Thus, we hypothesis that SDS may have positive effect on relieving the fatty liver in laying hens. Here, we used oleic acid (OA) [36- 
38], dietary energy, and protein structure recombination [39-42] to establish chicken fatty liver models in vitro and in vivo to explore the effects of SDS on lipid metabolism, proliferation, apoptosis, and mitochondrial function of fatty liver in chickens.

\section{Materials And Methods}

\section{Purity determination of SDS}

The SDS was purchased from Nanjing Herb-Source Biotechnology Co. Ltd (Nanjing, China). Its purity and structure were examined via High Performance Liquid Chromatography (HPLC) (Agilent Technologies Inc., California, USA), Nuclear Magnetic Resonance Spectrometer (NMR) (Avance III HD 400, Bruker, Switzerland), and Mass Spectrometry (MS) (Thermo Fisher Scientific, San Jose, CA).

\section{Animals}

A total of three hundred 35-week-old Rohman layers were raised at the Animal Breeding Farm, Sichuan Agricultural University (Ya'an, China). All animal experiments were approved by the Institutional Animal Care and Use Committee of Sichuan Agricultural University (Certification No. YCS-B2018102013). All experiments were conducted in accordance with the Laboratory Animal Welfare and Ethics guidelines of Sichuan Agricultural University.

\section{Cell culture and treatment}

Primary hepatocytes were isolated from the Rohman layers according to the methods described by [43, 44]. The hepatocytes were resuspended in William's medium E (Sigma, Shanghai, China) and plated into 6-well plates $\left(2 \times 10^{6}\right.$ cells/well) after cell counting. The hepatocytes were cultured in a cell culture incubator at a constant temperature of $37^{\circ} \mathrm{C}, 5 \% \mathrm{CO}_{2}$, and $95 \%$ air saturated humidity. After $24 \mathrm{~h}$, the hepatocytes were cultured with $0.6 \mathrm{mM} \mathrm{OA}$ for $12,24,36$ and $48 \mathrm{~h}$ and treated with/without SDS. All hepatocytes were collected for further experiments.

\section{RNA extraction and quantitative real time PCR (qRT-PCR)}

Total RNA was extracted from all samples according to the instructions of the Trizol reagent (Molecular Research Center, Cincinnati, $\mathrm{OH}$ ). The RNA concentration and purity were estimated by determining the A260/A280 absorbance ratio, and the $18 \mathrm{~S}$ and $28 \mathrm{~S}$ bands in a $1 \%$ agarose gel. Reverse transcription and qRT-PCR were performed as described previously [45]. GAPDH and $\beta$-actin were used as endogenous controls to normalize gene expression using the $2^{-\Delta \Delta \mathrm{Ct}}$ method [46]. Gene-specific primers designed with software Primer Premier 5.0 according to the coding sequences of target genes are summarized in Supplementary table 1.

\section{Protein extraction and western blot analysis}

Hepatocyte protein was isolated using a protein extraction kit (BestBio Biotech Co. Ltd., Shanghai, China), and the BCA protein assay kit (BestBio) was used to determine the concentration of protein samples. 
Western blotting was performed with the method described by [47] with the following primary antibodies: anti-PPARa (Abcam, Cambridge, UK), anti-PCNA (ABclonal Technology, Wuhan, China), anti-CDK2 (ZenBio, Chengdu, Chin), anti-caspase-3 and caspase-8 (Abcam), and caspase-9 (Bioss, Beijing, China), antiPI3K [Cell Signaling Technology (CST), USA], anti-p-PI3K (CST), anti-AKT and p-AKT (CST), and anti-Gsk3$\beta$ and $p$-Gsk3- $\beta$ (ABclonal). Goat anti-mouse and goat anti-rabbit secondary antibodies (Zen-Bio) $\beta$ Tubulin (Zen-Bio) was used as a reference.

\section{Staining for liver tissue and hepatocyte}

Liver tissues $(\mu \mathrm{m} / \mathrm{g}$ ) were fixed with $4 \%$ paraformaldehyde for $24 \mathrm{~h}$ and dehydrated with different concentrations of alcohol. Thereafter, the tissue mass was embedded in paraffin, cut into thin slices and placed on a slide. After hematoxylin and eosin (HE) staining, the sections were sealed with neutral resin. Liver tissue was sampled as a size of $24 \mathrm{~mm} \times 24 \mathrm{~mm} \times 2 \mathrm{~mm}$, frozen and then placed on a tissue bearer dripping with an OTC embedding agent. Slices (thickness $10 \mu \mathrm{m}$ ) were obtained with the slicing machine (Leica CM1520, Germany) and affixed to the anti-slip slide. After staining with oil red $\mathrm{O}$ staining solution for $15 \mathrm{~min}$, the slices were sealed with a neutral resin.

Hepatocyte Oil red 0 staining was performed following the manufacturers' protocol. Briefly, hepatocytes were washed with phosphate buffered saline (PBS) and fixed with ORO Fixative for $30 \mathrm{~min}$, and then the stationary fluid was discarded and washed with PBS twice. Thereafter, $60 \%$ isopropanol was added and maintained for $5 \mathrm{~min}$, after which the ORO Stain was added and retained for $20 \mathrm{~min}$. After washing 5 times, the Mayer Hematoxylin staining solution was added and kept for $2 \mathrm{~min}$. Hepatocytes were washed and ORO Buffer was added and sustained for $1 \mathrm{~min}$. After washing with PBS, the hepatocytes were covered with distilled water. All sections and hepatocytes were viewed under a microscope (DP80Digital, Olympus, Tokyo, Japan) and ten fields were randomly selected for statistical analysis.

\section{Cell counting kit-8 (CCK-8) and 5-ethynyl-2-deoxyuridine (EdU) assay}

The CCK-8 Kit (MeilunBio, Dalian, China) was used to test hepatocyte activity and choose the optimum concentration and treatment time of SDS in the OA-induced fatty liver model of primary hepatocytes. Ten $\mu \mathrm{L}$ of CCK-8 reagent was added to each well and incubated in a cell culture incubator for $2 \mathrm{~h}$ after being treated with SDS for 12, 24, 36, and 48 h. A microplate reader (Varioskan LUX, Thermo Fisher, USA) was used to determine the optical density (OD) of each sample at $450 \mathrm{~nm}$. The proliferation state of hepatocytes was determined using a Cell-Light ${ }^{\text {TM }}$ EdU kit (RiboBio, Guangzhou, China) according to the manufacturer's instructions. A fluorescent microscope was used to calculate the number of EdU-positive cells.

\section{Cell apoptosis and reactive oxygen species (ROS) analysis}

Hepatocyte apoptosis was detected using Flow Cytometry (CytoFLEX, Beckman, USA) and Kaluza 2.1 software. Hepatocytes were washed with PBS and the concentration was adjusted to $1 \times 10^{6} \mathrm{cells} / \mathrm{mL}$, and determination was performed as previously described [48]. DCFH-DA (2, 7-Dichlorofluorescin; Sigma) 
was added to hepatocytes at a final concentration of $10 \mu \mathrm{M}$ for 20 min at room temperature and hepatocyte ROS were analyzed using a Shimadzu RF-3501 fluorescence spectrophotometer at an excitation wavelength of $488 \mathrm{~nm}$ and an emission wavelength of $525 \mathrm{~nm}$.

\section{$\mathrm{PIBK} / \mathrm{AKT} / \mathrm{Gsk} 3-\beta$ pathway analysis}

LY294002 (Houston, Texas, United States) was used as a PI3K inhibitor to explore whether SDS exerts its biological function through PI3K/AKT/Gsk3- $\beta$. LY294002 was purchased from Selleck Chemicals and preincubated with hepatocytes for $2 \mathrm{~h}$.

\section{In vivo experiments in laying hens}

In Trial 1, we wanted to find out the optimal feeding concentration of SDS. Thus, 180 35-week-old Rohman layers were randomly divided into six groups, including the Control (Con) and SDS feeding groups $(5,10,20,40$, and $80 \mathrm{mg} / \mathrm{kg}$, respectively). Additive feeding experiment lasted for 4 weeks. Serum biochemical parameters including total cholesterol (TC), Triglyceride (TG), alanine aminotransferase (ALT), aspartate aminotransferase (AST), and superoxide dismutase (SOD) were measured with enzymelinked immunosorbent assay (ELISA) kits (Baolai Biotechnology Co., Ltd, Yancheng, China), following the manufacturer's instructions. Moreover, the expression abundances of lipid metabolism-related genes and inflammatory factors were detected using qRT-PCR.

In Trial 2, our purpose was to make clear whether SDS can prevent fatty liver in laying birds. Thus, 144 35week-old Rohman layers were adopted and separated into four groups: Con, Con + SDS, Model, and Model + SDS. Based on Trial 1, optimal feeding dosage of SDS $(20 \mathrm{mg} / \mathrm{kg})$ was used to feed the birds for 4 weeks in group Con + SDS and Model + SDS. The fatty liver model (Model) of laying hens in vivo was established with the method previously described by [39-42]. The diet composition was summarized in Supplementary table 2 .

\section{Statistical analysis}

The statistical analyses were carried out using SAS 9.3 software (SAS Institute Inc., Cary, NC, USA), and the mean of three replicates were evaluated and presented as mean \pm standard error (SE). Significance level was determined using Duncan's multiple range tests and displayed as $P<0.05\left(^{\star}\right)$ and $P<0.01(* \star)$.

\section{Results}

\section{Purity determination of SDS}

The purity and structure of SDS $\left(\mathrm{C}_{14} \mathrm{H}_{20} \mathrm{O}_{7}, 300.30\right.$, Fig. $\left.1 \mathrm{~A}\right)$ were detected using HPLC, NMR, and MS. The HPLC analysis results showed that the area reached a peak at a run time of $14.196 \mathrm{~min}$, with a peak area rate of $99.81 \%$ (Fig. 1B). NMR analysis results showed that the structure complied with the molecular 
formula of $\mathrm{C}_{14} \mathrm{H}_{20} \mathrm{O}_{7}$ (Fig. 1C). MS analysis showed that the structure complied with the molecular weight of $300.30 \mathrm{Da}$ (Fig. 1D).

\section{SDS treatment with OA-induced fatty liver model in primary hepatocytes}

OA $(0.6 \mathrm{mM})$ was used for inducing fatty liver model in primary hepatocytes. Cells were treated with 0.1 , $0.2,0.3,0.4 \mathrm{mg} / \mathrm{mL}$ SDS for $48 \mathrm{~h}$, respectively (Supplementary table 3 ). According to the OD values of the CCK-8 assay at $12,24,36$, and $48 \mathrm{~h}, \mathrm{OA}$ significantly decreased hepatocyte activity compared with the control, while SDS significantly increased hepatocyte activity at the concentration of $0.3 \mathrm{mg} / \mathrm{mL}$ at $36 \mathrm{~h}$ (Fig. 2).

\section{SDS inhibits OA-induced lipid accumulation in chicken primary hepatocytes}

Oil red $O$ staining was used to test lipid accumulation and the results showed that numerous lipid droplets appeared in the OA group. SDS significantly decreased OA-induced lipid accumulation in hepatocytes (Fig. 3A). In addition, the mRNA abundance and protein level of peroxisome proliferatoractivated a (PPARa) significantly decreased in the OA group, whereas SDS significantly increased the mRNA and protein levels of PPARa (Fig. 3B and C). The mRNA expression of acyl-CoA synthetase longchain 1 (ACSL 1) and microsomal triglyceride transport protein (MTTP) were decreased in the OA group. However, SDS increased their mRNA expression levels in group OA+SDS. The mRNA expression abundances of peroxisome proliferator-activated $\mathrm{Y}(P P A R Y)$, fatty acid synthase (FASN), Fatty acid desaturase 1 (FADS1), and fatty acid desaturase 2 (FADS2) were significantly increased in the OA group while SDS decreased their mRNA expression levels in group OA + SDS (Fig. 3B).

\section{SDS promotes hepatocyte proliferation in OA-induced fatty liver model}

We investigated the effect of SDS on chicken hepatocyte proliferation in an OA-induced fatty liver model. We found that OA resulted in a decrease in the mRNA and protein levels of proliferation related genes [cyclin-dependent kinase 2 (CDK2) and proliferating cell nuclear antigen (PCNA)], and decreased the mRNA expression of cyclinD1. SDS significantly increased the mRNA expression of $P C N A, C D K 2$, and cyclinD1, as well as the protein expression level of PCNA and CDK2 (Fig. 4A, B and C). The EdU assay revealed a decrease in the number of proliferating cells in response to treatment with $O A$, while the cell proliferation rate significantly increased in the SDS treatment group (Fig. 4D and E).

\section{SDS inhibits hepatocyte apoptosis in OA-induced fatty liver model}

We further investigated whether SDS influenced hepatocyte apoptosis in OA-induced fatty liver and found that OA significantly increased the mRNA expression of Caspase-8, Caspase-9, and Caspase-3, and protein expression levels of Caspase-9 and Caspase-3, didn't affect the protein expression of Caspase-8. 
However, the mRNA expression and cleavage levels of Caspase-9, Caspase-8, and Caspase- 3 were significantly decreased in the SDS treatment group (Fig. 5A, B, and C). Excessive ROS production and mitochondrial dysfunction lead to liver steatosis and hepatocyte apoptosis $[49,50]$. We further examined the effect of SDS on hepatocyte ROS levels in the OA-induced fatty liver model. The results showed that OA led to an increase in hepatocyte mitochondrial ROS production, while SDS significantly decreased the ROS level compared to the NC group (Fig. 5D and E). An increased number of early and necrotic late apoptosis hepatocytes appeared in the OA-induced fatty liver group, and SDS significantly decreased both early and necrotic late apoptosis in hepatocytes compared with the NC group (Fig. 5F and G).

\section{SDS targets the PI3K/AKT/Gsk3- $\beta$ pathway}

Phosphoinositide 3-kinase (PI3K)/AKT-glycogen synthase kinase 3 beta (Gsk3- $\beta$ ) is an important antiapoptotic signaling pathway that activates a series of growth signaling pathways and blocks a series of apoptotic signaling pathways, thus promoting cell survival and proliferation [51, 52]. In the present study, we attempted to uncover the function and regulation pathways of SDS in an OA-induced fatty liver model. The results showed that protein levels of $p-P D K 1, p-A K T$, and $p-G s k 3-\beta$ in the OA treated group were lower than those in the NC group $(P<0.05)$, while the SDS $(O A+$ Salidroside) treatment group had higher protein levels than the OA treatment group $(P<0.05)$. Moreover, we added LY294002 as a PI3K inhibitor in the SDS treatment group, and found that the protein levels of p-PDK1, p-AKT, and p-Gsk3- $\beta$ in the PI3K inhibitor (OA + Salidroside + LY294002) treated group were lower than those in the SDS treatment group (Fig. 6A and B) $(P<0.05)$. Generally, SDS inhibits lipid accumulation and hepatocyte apoptosis, and promotes hepatocyte proliferation in an OA-induced fatty liver model targeting the PI3K/AKT/Gsk3- $\beta$ pathway.

\section{SDS alleviates hepatic steatosis and inflammatory response of laying hens in vivo}

In Trail 1, we found that TC, TG, ALT, and AST levels in the serum of SDS feeding groups were decreased, compared with the Control group (Con). The TC concentration was significantly decreased at the level 10, 20 , and $40 \mathrm{mg} / \mathrm{kg}$ for SDS, and reached the bottom at the level $20 \mathrm{mg} / \mathrm{kg}(P<0.05)$. The AST level was significantly decreased in all SDS concentration groups, compared with the control $(P<0.05)$. There were no significant differences for SOD levels among the groups (Fig. 7). Moreover, compared to the Con group, the mRNA expression abundance of PPARa was significantly increased in all SDS treatment groups $(5,10,20,40$, and $80 \mathrm{mg} / \mathrm{kg})(P<0.05)$. The mRNA expression of $P P A R Y$ significantly decreased in the groups 40 and $80 \mathrm{mg} / \mathrm{kg}$ SDS $(P<0.05)$. The mRNA expression of MTTP was significantly increased in all the SDS treatment groups. The mRNA expression of FASN was significantly decreased at the groups $5,20,40$ and $80 \mathrm{mg} / \mathrm{kg}$ SDS $(P<0.05)$. The mRNA levels of the inflammatory factors $I L-6$ and $I L-1 \beta$ were significantly decreased in the groups 20,40 and $80 \mathrm{mg} / \mathrm{kg}$ SDS $(P<0.05)$ (Fig. 8).

Based on above results, optimal feeding concentration of SDS was verified as $20 \mathrm{mg} / \mathrm{kg}$. In Trial 2, we found that the body weight, liver weight, and abdominal fat weight were significantly increased in the 
Model group, and significantly decreased in the Con + SDS and Model + SDS group, compared with the Con group (Table 1). H \& E staining of the liver tissues in the Con group showed that the cytoplasm was lightly stained, and appeared a few small fatty vacuoles. Oil red $O$ staining showed that small lipid droplets appeared in the liver, which indicated mild steatosis. In the Model group we found a large number of fatty vacuoles, the hepatic cord was disorganized the sinuses were atretic, and oil red 0 staining showed that numerous lipid droplets appeared in the liver, which indicated severe steatosis. In group Con + SDS and Model + SDS, we observed that the hepatocytes were arranged neatly and clearly, the nucleus were at the center of the cell (blue) and the cytoplasm of the hepatocytes were evenly distributed (pink). Oil red O staining showed that both Con + SDS and Model + SDS groups had fewer fat droplets than the Con group (Fig. 9).

Table 1

Body, liver and abdominal fat weight measurements

\begin{tabular}{|lllll|}
\hline Weight (g) & Con & Con + SDS & Model & Model + SDS \\
\hline Body $(0 \mathrm{~d})$ & $1760 \pm 190$ & $1740 \pm 190$ & $1730 \pm 140$ & $1740 \pm 130$ \\
\hline Body $(28 \mathrm{~d})$ & $1770 \pm 110^{\mathrm{b}}$ & $1650 \pm 110^{\mathrm{c}}$ & $1920 \pm 150^{\mathrm{a}}$ & $1700 \pm 90^{\mathrm{c}}$ \\
\hline Liver & $50.67 \pm 7.52^{\mathrm{b}}$ & $33.53 \pm 3.62^{\mathrm{c}}$ & $58.72 \pm 6.88^{\mathrm{a}}$ & $26.17 \pm 1.82^{\mathrm{c}}$ \\
\hline Abdominal fat & $72.34 \pm 15.35^{\mathrm{b}}$ & $33.05 \pm 12.52^{\mathrm{c}}$ & $94.98 \pm 16.12^{\mathrm{a}}$ & $40.97 \pm 13.02^{\mathrm{c}}$ \\
\hline $\begin{array}{l}\text { Data was shown as the mean } \pm \text { standard error }(\mathrm{SE})(\mathrm{n}=9) \text {. Means within a line marked without the } \\
\text { same superscripts differed significantly }(P<0.05) .\end{array}$ & \\
\hline
\end{tabular}

Compared with the Con group, both TC and TG levels in serum were significantly increased in Model group and decreased in Con + SDS and Model + SDS groups $(P<0.05)$. The ALT and AST levels in group Con + SDS and Model + SDS were significantly lower than those in Model group $(P<0.05)$. Moreover, we found that SOD activity in group Con + SDS was higher than those in group Con and Model $(P<0.05$, Fig. 10).

Compared with the Con group, the mRNA expression abundances of PPARa and MTTP was significantly increased in the group Con + SDS and Model + SDS $(P<0.05)$. The mRNA expressions abundances of $P P A R Y, S C D$, and $F A S$ were significantly increased in the Model group while decreased in the Con + SDS and Model + SDS group $(P<0.05)$. Moreover, the mRNA expressions of TNF- $a, I L-1 \beta, I L-6$, and $I L-8$ were significantly increased in the Model group while decreased in the Con + SDS and Model + SDS groups ( $P$ $<0.05$ ) (Fig. 11). Our results suggest that SDS alleviates high-fat diet-induced hepatic steatosis, oxidative stress and the inflammatory response of laying hens in vivo.

\section{Discussion}

Fatty liver hemorrhagic syndrome (FLHS) is a chronic hepatic disease caused by a disorder of lipid metabolism, which usually presents as steatosis, cirrhosis, liver fibrosis, and NAFLD [53, 54]. FLHS occurs 
in cage laying hens with high frequency and is characterized by decreased egg production and unexplained death of laying hens. FLHS accounts for $74 \%$ of the total mortality of cage laying hens in Queensland, Australia [40]. In addition, FLHS is the most common cause of non-communicable chicken deaths in Northern California [55]. FLHS caused huge losses to the poultry industry.

SDS is a herbal drug which grows at high altitude areas [56] and have numerous pharmacological effects, e.g. protective effects on mitochondrial function [57], anti-apoptotic and anti-inflammatory effects [58], and antioxidant effects [59,60]. Multiple studies suggested that SDS could reduce the liver lipid accumulation in both the type 2 diabetic [34] and NAFLD mice [35]. In the current study, we found that SDS inhibited OA-induced lipid accumulation in primary chicken hepatocytes. Moreover, SDS promoted hepatocyte proliferation and inhibited its apoptosis in an OA-induced fatty liver model. SDS increased the hepatocyte activity and the mRNA expression of proliferation related genes PCNA, CDK2, and cyclinD1, and the protein expression levels of PCNA and CDK2. Moreover, SDS decreased the cleavage levels of Caspase- 9 , Caspase-8, and Caspase-3, and also the hepatocytes apoptosis. These results were consistent with the previous studies which indicated that SDS increased the protein expression of cyclin-dependent kinases (CDKs) [61] and Cyclin D1 [62], and suppressed cell apoptosis by inhibiting the pro-apoptotic protein expression of cleaved-Caspase-3/9 [62]. Our study showed that SDS significantly attenuated OA-induced ROS generation, which was consistent with the finding that SDS attenuated high-fat diet-induced ROS generation in NAFLD mice [35].

$\mathrm{PI} 3 \mathrm{~K} / \mathrm{AKT} / \mathrm{Gsk} 3-\beta$ is a critical anti-apoptotic signaling pathway which activates a series of growth signaling pathways and blocks apoptotic signaling pathways, thereby promoting cell survival and proliferation [51, 52]. Zhang et al. suggested that SDS protected against 1-methyl-4-phenylpyridineinduced cell apoptosis in part by regulating the PI3K/AKT/Gsk3- $\beta$ pathway. SDS increased the phosphorylation levels of AKT and Gsk3- $\beta$, and inhibited the activation of caspase-3, caspase- 6 , and caspase-9 [52]. SDS dose-dependently increased the phosphorylation of the mitochondria-associated $\mathrm{PI} 3 \mathrm{~K} / \mathrm{AKT} / \mathrm{Gsk} 3-\beta$ pathway in hepatocytes [63], and alleviated sepsis induced myocarditis in rats by regulating the PI3K/AKT/Gsk3- $\beta$ signaling pathway [64]. In the present study, we found that SDS increased the phosphorylation levels of PDK1, AKT, and Gsk3- $\beta$ but decreased the PI3K inhibitor. We determined that SDS alleviated lipid accumulation, hepatocyte apoptosis, and promoted hepatocyte proliferation in the OA-induced fatty liver model by targeting the PI3K/AKT/Gsk3- $\beta$ pathway.

Furthermore, we investigated the effects of SDS on HFD-induced FLHS in laying hens in vivo. Results indicated that the layer's body weight, liver weight, and abdominal fat weight were significantly increased in the Model group which having severe steatosis, whereas all those traits were decreased and improved in SDS feeding groups. Additionally, high-fat diet-induced FLHS of laying hens showed the high levels of TC, TG, ALT, and AST in serum, while SDS improved all those indexes and increased SOD activity of highfat diet-fed birds. Zheng et al. detected that SDS alleviated hepatic steatosis, oxidative stress, and inflammatory reactions in the liver of NAFLD mice, characterized by the decreased levels of TC, TG, ALT, and AST in serum, inhibition of hepatic lipid deposition and the gene expression of FAS, and suppression of the gene expression of TNF- $a$ and $I L-1 \beta$ in the liver [35]. Moreover, Amevor et al. [65] reported there were 
decreased levels of AST and ALT in serum, and no liver steatosis in chickens fed with the diets containing both dietary quercetin and vitamin $\mathrm{E}$ (dietary antioxidants). The increased expression of lipogenesis gene FAS accelerated the ectopic deposition of TG [66]. In the current study, we found that SDS decreased the liver mRNA expression of PPARY, $S C D$, and $F A S$, whereas increased the mRNA expression of PPARa and MTTP in high-fat diet-induced FLHS of laying hens in vivo. Inflammatory cytokine TNF-a, IL-6, IL-8, and IL$1 \beta$ played important roles in the inflammatory response [67]. Previous studies reported that SDS alleviated cell injury and lipid accumulation, and inhibited the mRNA expression of $I L-1 \beta$ and $I L-6$ in human NAFLD [68], alleviated LPS-induced injury in humans by decreasing inflammatory chemokines IL6 and TNF-a [62], and improved the survival rate of endotoxemia mice by blocking the activation of NF-KB and inhibiting the expression and release of inflammatory cytokines TNF- $a$, IL-6, and IL-1 $\beta$ [69]. We found that SDS decreased the mRNA expression of TNF- $a, I L-1 \beta, I L-6$, and $I L-8$. These results indicate that SDS alleviates HFD-induced hepatic steatosis, oxidative stress, and the inflammatory response of laying hens in vivo.

\section{Conclusions}

Oleic acid (OA)-induced fatty liver model and high-fat diet-induced FLHS were successfully built in vitro and in vivo in the present study. SDS attenuated OA-induced ROS generation, inhibited lipid accumulation and hepatocyte apoptosis, and promoted hepatocyte proliferation in the OA-induced fatty liver model in vitro by targeting the PI3K/AKT/Gsk3- $\beta$ pathway. Moreover, SDS alleviated high-fat diet-induced hepatic steatosis, oxidative stress, and the inflammatory response of laying hens in vivo.

\section{Abbreviations}

ACSL1, acyl-CoA synthetase long-chain 1; ALT, alanine transaminase; AST, aspartate aminotransferase; CDK2, cyclin-dependent kinase 2; FADS1, Fatty acid desaturase 1; FASN, fatty acid synthase; FLHS, fatty liver hemorrhagic syndrome; Gsk3- $\beta$, glycogen synthase kinase 3 beta; HPLC, High Performance Liquid Chromatography; MS, Mass Spectrometry; MTTP, microsomal triglyceride transport protein; NMR, Nuclear Magnetic Resonance Spectrometer; OA, oleic acid; PCNA, proliferating cell nuclear antigen; PI3K, Phosphoinositide 3-kinase; PPARa, peroxisome proliferator-activated a; ROS, reactive oxygen species; SDS, salidroside; SOD, superoxide dismutase; TC, total cholesterol; TG, triglyceride.

\section{Declarations}

\section{Authors' contributions}

ZC wrote the paper draft; FA and XZ corrected the draft; XZ, GS and JF supervised the experimentators; $Z C$ and NJ performed the experiments. ZC, LL, XD, XK, DX, ZN, XD, and WS analyzed the data; ZC, YT, QZ, $Y W, D L, Y Z, X W$, and $X H$ generated the images. 
All data were generated in-house, and no paper mill was used. All authors agree to be accountable for all aspects of work ensuring integrity and accuracy.

\section{Funding}

The authors thank the Sichuan Science and Technology Program (2020JDRC0104), the Key Research \& Development Plan of the Department of Science and Technology of Tibet Autonomous Region (XZ202101ZY0002N), the Local Projects Guided by the Central Government from Razi County, Tibet Autonomous Region, and the Projects Funded by the Central Government to Guide Local Scientific and Technological Development from Guizhou province ([2021]4003) for funding this work.

\section{Informed Consent Statement}

Not applicable.

\section{Data Availability Statement}

All data presented in this study are available on request from the corresponding author.

\section{Ethics approval and consent to participate}

All animal experiments were approved by the Institutional Animal Care and Use Committee of Sichuan Agricultural University (Certification No. YCS-B2018102013). All experiments were conducted in accordance with the Laboratory Animal Welfare and Ethics guidelines of Sichuan Agricultural University.

\section{Consent for publication}

All the authors read and agree to the content of this paper and its publication.

\section{Conflict of interest}

The authors have declared that no competing interest exists.

\section{References}

1. Jensen LS, Casey JM, Savage SI, Britton WM. An association of hardness of water with incidence of fatty liver syndrome in laying hens. Poult Sci. 1976;55(2):719-24.

2. Wolford JH, Polin D. Lipid accumulation and hemorrhage in livers of laying chickens. A study on fatty liver-hemorrhagic syndrome (FLHS). Poult Sci. 1972;51(5):1707-13.

3. Neuschwander-Tetri BA. Fatty liver and the metabolic syndrome. Curr Opin Gastroenterol. 2007;23(2):193-8.

4. Squires EJ, Leeson S. Aetiology of fatty liver syndrome in laying hens. Br Vet J. 1988;144(6):602-9. 
5. Koek GH, Liedorp PR, Bast A. The role of oxidative stress in non-alcoholic steatohepatitis. Clin Chim Acta. 2011;412(15-16):1297-305.

6. Shini A, Shini S, Bryden WL. Fatty liver haemorrhagic syndrome occurrence in laying hens: impact of production system. Avian Pathol. 2019;48(1):25-34. .

7. Butler EJ. Fatty liver diseases in the domestic fowl--a review. Avian Pathol. 1976;5(1):1-14.

8. Egnatchik RA, Leamy AK, Noguchi Y, Shiota M, Young JD. Palmitate-induced activation of mitochondrial metabolism promotes oxidative stress and apoptosis in H4IIEC3 rat hepatocytes. Metabolism. 2014;63(2):283-95.

9. Wang LJ, Zhang HW, Zhou JY, Liu Y, Yang Y, Chen XL, Zhu CH, Zheng RD, Ling WH, Zhu HL. Betaine attenuates hepatic steatosis by reducing methylation of the MTTP promoter and elevating genomic methylation in mice fed a high-fat diet. J Nutr Biochem. 2014;25(3):329-36.

10. Koo SH. Nonalcoholic fatty liver disease: molecular mechanisms for the hepatic steatosis. Clin Mol Hepatol. 2013;19(3):210-5.

11. Reccia I, Kumar J, Akladios C, Virdis F, Pai M, Habib N, Spalding D. Non-alcoholic fatty liver disease: A sign of systemic disease. Metabolism. 2017;72:94-108.

12. Mantena SK, King AL, Andringa KK, Eccleston HB, Bailey SM. Mitochondrial dysfunction and oxidative stress in the pathogenesis of alcohol- and obesity-induced fatty liver diseases. Free Radic Biol Med. 2008;44(7):1259-72.

13. Feng X, Yu W, Li X, Zhou F, Zhang W, Shen Q, Li J, Zhang C, Shen P. Apigenin, a modulator of PPARY, attenuates HFD-induced NAFLD by regulating hepatocyte lipid metabolism and oxidative stress via Nrf2 activation. Biochem Pharmacol. 2017;136:136-49.

14. Wang X, Xing C, Yang F, Zhou S, Li G, Zhang C, Cao H, Hu G. Abnormal expression of liver autophagy and apoptosis-related mRNA in fatty liver haemorrhagic syndrome and improvement function of resveratrol in laying hens. Avian Pathol. 2020;49(2):171-8.

15. Huang WC, Chen YL, Liu HC, Wu SJ, Liou CJ. Ginkgolide C reduced oleic acid-induced lipid accumulation in HepG2 cells. Saudi Pharm J. 2018;26(8):1178-84.

16. Lu L, Yuan J, Zhang S. Rejuvenating activity of salidroside (SDS): dietary intake of SDS enhances the immune response of aged rats. Age (Dordr). 2013;35(3):637-46.

17. Lv C, Huang Y, Liu ZX, Yu D, Bai ZM. Salidroside reduces renal cell carcinoma proliferation by inhibiting JAK2/STAT3 signaling. Cancer Biomark. 2016;17(1):41-7.

18. Yang ZR, Wang HF, Zuo TC, Guan LL, Dai N. Salidroside alleviates oxidative stress in the liver with non- alcoholic steatohepatitis in rats. BMC Pharmacol Toxicol. 2016;17:16.

19. Dhar P, Bajpai PK, Tayade AB, Chaurasia OP, Srivastava RB, Singh SB. Chemical composition and antioxidant capacities of phytococktail extracts from trans-Himalayan cold desert. BMC Complement Altern Med. 2013;13:259.

20. Yuan Y, Wu SJ, Liu X, Zhang LL. Antioxidant effect of salidroside and its protective effect against furan-induced hepatocyte damage in mice. Food Funct. 2013;4(5):763-9. 
21. Yu P, Hu C, Meehan EJ, Chen L. X-ray crystal structure and antioxidant activity of salidroside, a phenylethanoid glycoside. Chem Biodivers. 2007;4(3):508-13.

22. Zhu J, Wan X, Zhu Y, Ma X, Zheng Y, Zhang T. Evaluation of salidroside in vitro and in vivo genotoxicity. Drug Chem Toxicol. 2010;33(2):220-6.

23. Mao GX, Deng HB, Yuan LG, Li DD, Li YY, Wang Z. Protective role of salidroside against aging in a mouse model induced by D-galactose. Biomed Environ Sci. 2010;23(2):161-6.

24. Zhang L, Yu H, Sun Y, Lin X, Chen B, Tan C, Cao G, Wang Z. Protective effects of salidroside on hydrogen peroxide-induced apoptosis in SH-SY5Y human neuroblastoma cells. Eur $\mathrm{J}$ Pharmacol. 2007;564(1-3):18-25.

25. Guan S, Xiong Y, Song B, Song Y, Wang D, Chu X, Chen N, Huo M, Deng X, Lu J. Protective effects of salidroside from Rhodiola rosea on LPS-induced acute lung injury in mice. Immunopharmacol Immunotoxicol. 2012;34(4):667-72.

26. Li D, Fu Y, Wen Z. Salidroside attenuates inflammatory responses by suppressing nuclear factor-kB and mitogen activated protein kinases activation in lipopolysaccharide-induced mastitis in mice. Inflamm Res. 2013;62(1):9-15.

27. Wu YL, Lian LH, Jiang YZ, Nan JX. Hepatoprotective effects of salidroside on fulminant hepatic failure induced by D-galactosamine and lipopolysaccharide in mice. J Pharm Pharmacol. 2009;61(10):1375-82.

28. Wu YL, Piao DM, Han XH, Nan JX. Protective effects of salidroside against acetaminophen-induced toxicity in mice. Biol Pharm Bull. 2008;31(8):1523-9.

29. Zuo G, Li Z, Chen L, Xu X. Activity of compounds from Chinese herbal medicine Rhodiola kirilowii (Regel) Maxim against HCV NS3 serine protease. Antiviral Res. 2007;76(1):86-92.

30. Xiong Y, Wang Y, Xiong Y, Gao W, Teng L. Salidroside alleviated hypoxia-induced liver injury by inhibiting endoplasmic reticulum stress-mediated apoptosis via IRE1a/JNK pathway. Biochem Biophys Res Commun. 2020;529(2):335-40.

31. Lin SY, Dan X, Du XX, Ran CL, Lu X, Ren SJ, Tang ZT, Yin LZ, He CL, Yuan ZX, Fu HL, Zhao XL, Shu G. Protective Effects of Salidroside against Carbon Tetrachloride (CCl4)-Induced Liver Injury by Initiating Mitochondria to Resist Oxidative Stress in Mice. Int J Mol Sci. 2019;20(13):3187.

32. Feng J, Niu P, Chen K, Wu L, Liu T, Xu S, Li J, Li S, Wang W, Lu X, Yu Q, Liu N, Xu L, Wang F, Dai W, Xia $Y$, Fan X, Guo C. Salidroside mediates apoptosis and autophagy inhibition in concanavalin A-induced liver injury. Exp Ther Med. 2018 Jun;15(6):4599-614.

33. Li F, Tang H, Xiao F, Gong J, Peng Y, Meng X. Protective effect of salidroside from Rhodiolae Radix on diabetes-induced oxidative stress in mice. Molecules. 2011;16(12):9912-24.

34. Zhang XR, Fu XJ, Zhu DS, Zhang CZ, Hou S, Li M, Yang XH. Salidroside-regulated lipid metabolism with down-regulation of miR-370 in type 2 diabetic mice. Eur J Pharmacol. 2016;779:46-52.

35. Zheng T, Yang X, Li W, Wang Q, Chen L, Wu D, Bian F, Xing S, Jin S. Salidroside Attenuates High-Fat Diet-Induced Nonalcoholic Fatty Liver Disease via AMPK-Dependent TXNIP/NLRP3 Pathway. Oxid Med Cell Longev. 2018;2018:8597897. 
36. Li L, Chu X, Yao Y, Cao J, Li Q, Ma H. (-)-Hydroxycitric Acid Alleviates Oleic Acid-Induced Steatosis, Oxidative Stress, and Inflammation in Primary Chicken Hepatocytes by Regulating AMP-Activated Protein Kinase-Mediated Reactive Oxygen Species Levels. J Agric Food Chem. 2020;68(40):1122941.

37. Espe M, Xie S, Araujo P. development of a fatty liver model. Aquacult Nutr. 2019, 25.

38. Weijler AM, Schmidinger B, Kapiotis S, Laggner $\mathrm{H}$, Hermann M. Oleic acid induces the novel apolipoprotein $\mathrm{O}$ and reduces mitochondrial membrane potential in chicken and human hepatoma cells. Biochimie. 2018;147:136-42.

39. Zhuang Y, Xing C, Cao H, Zhang C, Luo J, Guo X, Hu G. Insulin resistance and metabonomics analysis of fatty liver haemorrhagic syndrome in laying hens induced by a high-energy low-protein diet. Sci Rep. 2019;9(1):10141.

40. Rozenboim I, Mahato J, Cohen NA, Tirosh O. Low protein and high-energy diet: a possible natural cause of fatty liver hemorrhagic syndrome in caged White Leghorn laying hens. Poult Sci. 2016;95(3):612-21.

41. Maurice DV, Jensen LS. Influence of diet composition on hepatic lipid accumulation and hemorrhages in caged layers. Poult Sci. 1978;57(4):989-97.

42. Yang F, Ruan J, Wang T, Luo J, Cao H, Song Y, Huang J, Hu G. Improving effect of dietary soybean phospholipids supplement on hepatic and serum indexes relevant to fatty liver hemorrhagic syndrome in laying hens. Anim Sci J. 2017;88(11):1860-9.

43. Zhou XD, Dong XF, Tong JM, Xu P, Wang ZM. High levels of vitamin E affect retinol binding protein but not CYP26A1 in liver and hepatocytes from laying hens. Poult Sci. 2012;91(5):1135-41.

44. Qi XL, Wang J, Yue HY, Wu SG, Zhang YN, Ni HM, Guo Y, Zhang HJ, Qi GH. Trans10, cis12-conjugated linoleic acid exhibits a stronger antioxidant capacity than cis9, trans11-conjugated linoleic acid in primary cultures of laying hen hepatocytes. Poult Sci. 2018;97(12):4415-24.

45. Cui Z, Amevor FK, Feng Q, Kang X, Song W, Zhu Q, Wang Y, Li D, Zhao X. Sexual Maturity Promotes Yolk Precursor Synthesis and Follicle Development in Hens via Liver-Blood-Ovary Signal Axis. Animals (Basel). 2020;10(12):2348.

46. Livak KJ, Schmittgen TD. Analysis of relative gene expression data using real-time quantitative PCR and the 2(-Delta Delta C(T)) Method. Methods. 2001;25(4):402-8.

47. Cui Z, Liu L, Kwame Amevor F, Zhu Q, Wang Y, Li D, Shu G, Tian Y, Zhao X. High Expression of miR204 in Chicken Atrophic Ovaries Promotes Granulosa Cell Apoptosis and Inhibits Autophagy. Front Cell Dev Biol. 2020;8:580072.

48. Cui Z, Shen X, Zhang X, Li F, Amevor FK, Zhu Q, Wang Y, Li D, Shu G, Tian Y, Zhao X. A functional polymorphism of inhibin alpha subunit at miR-181b-1-3p-binding site regulates proliferation and apoptosis of chicken ovarian granular cells. Cell Tissue Res. 2021;384(2):545-60.

49. Liu GY, Sun YZ, Zhou N, Du XM, Yang J, Guo SJ. 3,3'-OH curcumin causes apoptosis in HepG2 cells through ROS-mediated pathway. Eur J Med Chem. 2016;112:157-63. 
50. Prieto I, Monsalve M. ROS homeostasis, a key determinant in liver ischemic-preconditioning. Redox Biol. 2017;12:1020-5.

51. Pap M, Cooper GM. Role of glycogen synthase kinase-3 in the phosphatidylinositol 3-Kinase/Akt cell survival pathway. J Biol Chem. 1998;273(32):19929-32.

52. Zhang W, He H, Song H, Zhao J, Li T, Wu L, Zhang X, Chen J. Neuroprotective Effects of Salidroside in the MPTP Mouse Model of Parkinson's Disease: Involvement of the PI3K/Akt/GSK3 $\beta$ Pathway. Parkinsons Dis. 2016;2016:9450137.

53. McCullough AJ. The clinical features, diagnosis and natural history of nonalcoholic fatty liver disease. Clin Liver Dis. 2004;8(3):521-33.

54. Cheng S, Liang S, Liu Q, Deng Z, Zhang Y, Du J, Zhang Y, Li S, Cheng B, Ling C. Diosgenin prevents high-fat diet-induced rat non-alcoholic fatty liver disease through the AMPK and LXR signaling pathways. Int J Mol Med. 2018;41(2):1089-95.

55. Mete A, Giannitti F, Barr B, Woods L, Anderson M. Causes of mortality in backyard chickens in northern California: 2007-2011. Avian Dis. 2013;57(2):311-5.

56. Booker A, Jalil B, Frommenwiler D, Reich E, Zhai L, Kulic Z, Heinrich M. The authenticity and quality of Rhodiola rosea products. Phytomedicine. 2016;23(7):754-62.

57. Zhang W, Peng M, Yang Y, Xiao Z, Song B, Lin Z. Protective Effects of Salidroside on Mitochondrial Functions against Exertional Heat Stroke-Induced Organ Damage in the Rat. Evid Based Complement Alternat Med. 2015;2015:504567.

58. Zhu L, Wei T, Gao J, Chang X, He H, Luo F, Zhou R, Ma C, Liu Y, Yan T. The cardioprotective effect of salidroside against myocardial ischemia reperfusion injury in rats by inhibiting apoptosis and inflammation. Apoptosis. 2015;20(11):1433-43.

59. Xiao L, Li H, Zhang J, Yang F, Huang A, Deng J, Liang M, Ma F, Hu M, Huang Z. Salidroside protects Caenorhabditis elegans neurons from polyglutamine-mediated toxicity by reducing oxidative stress. Molecules. 2014;19(6):7757-69.

60. Li X, Sipple J, Pang Q, Du W. Salidroside stimulates DNA repair enzyme Parp-1 activity in mouse HSC maintenance. Blood. 2012;119(18):4162-73.

61. Mao GX, Xing WM, Wen XL, Jia BB, Yang ZX, Wang YZ, Jin XQ, Wang GF, Yan J. Salidroside protects against premature senescence induced by ultraviolet $B$ irradiation in human dermal fibroblasts. Int $J$ Cosmet Sci. 2015;37(3):321-8.

62. Tian F, Chen Z, Zhang Y, Jiang J, Li T. Salidroside protects LPS-induced injury in human thyroid follicular epithelial cells by upregulation of MiR-27a. Life Sci. 2018;213:1-8.

63. Zheng T, Yang X, Wu D, Xing S, Bian F, Li W, Chi J, Bai X, Wu G, Chen X, Zhang Y, Jin S. Salidroside ameliorates insulin resistance through activation of a mitochondria-associated AMPK/PI3K/Akt/GSK3ß pathway. Br J Pharmacol. 2015;172(13):3284-301.

64. He H, Chang X, Gao J, Zhu L, Miao M, Yan T. Salidroside Mitigates Sepsis-Induced Myocarditis in Rats by Regulating IGF-1/PI3K/Akt/GSK-3ß Signaling. Inflammation. 2015;38(6):2178-84. 
65. Amevor FK, Cui Z, Du X, Ning Z, Shu G, Jin N, Deng X, Tian Y, Zhang Z, Kang X, Xu D, You G, Zhang Y, Li D, Wang Y, Zhu Q, Zhao X. Combination of Quercetin and Vitamin E Supplementation Promotes Yolk Precursor Synthesis and Follicle Development in Aging Breeder Hens via Liver-Blood-Ovary Signal Axis. Animals (Basel). 2021;11(7):1915.

66. Foretz M, Carling D, Guichard C, Ferré P, Foufelle F. AMP-activated protein kinase inhibits the glucoseactivated expression of fatty acid synthase gene in rat hepatocytes. J Biol Chem. 1998;273(24):14767-71.

67. Jain SK, Rains J, Croad J, Larson B, Jones K. Curcumin supplementation lowers TNF-alpha, IL-6, IL-8, and MCP-1 secretion in high glucose-treated cultured monocytes and blood levels of TNF-alpha, IL-6, MCP-1, glucose, and glycosylated hemoglobin in diabetic rats. Antioxid Redox Signal. 2009;11(2):241-9.

68. Feng Q, Liu C, Gao W, Geng XL, Dai N. Salidroside-Mitigated Inflammatory Injury of Hepatocytes with Non-Alcoholic Fatty Liver Disease via Inhibition TRPM2 Ion Channel Activation. Diabetes Metab Syndr Obes. 2019;12:2755-63.

69. Guan S, Feng H, Song B, Guo W, Xiong Y, Huang G, Zhong W, Huo M, Chen N, Lu J, Deng X. Salidroside attenuates LPS-induced pro-inflammatory cytokine responses and improves survival in murine endotoxemia. Int Immunopharmacol. 2011;11(12):2194-9.

\section{Figures}


b High Performance Liquid Chromatography, HPLC

a<smiles>OC[C@H]1OC(OCCc2ccc(O)cc2)[C@H](O)[C@H](O)[C@H]1O</smiles>

Molecular Formula (M. F.): $\mathrm{C}_{14} \mathrm{H}_{20} \mathrm{O}_{7}$

Molecular Weight (M. W.): 300.30
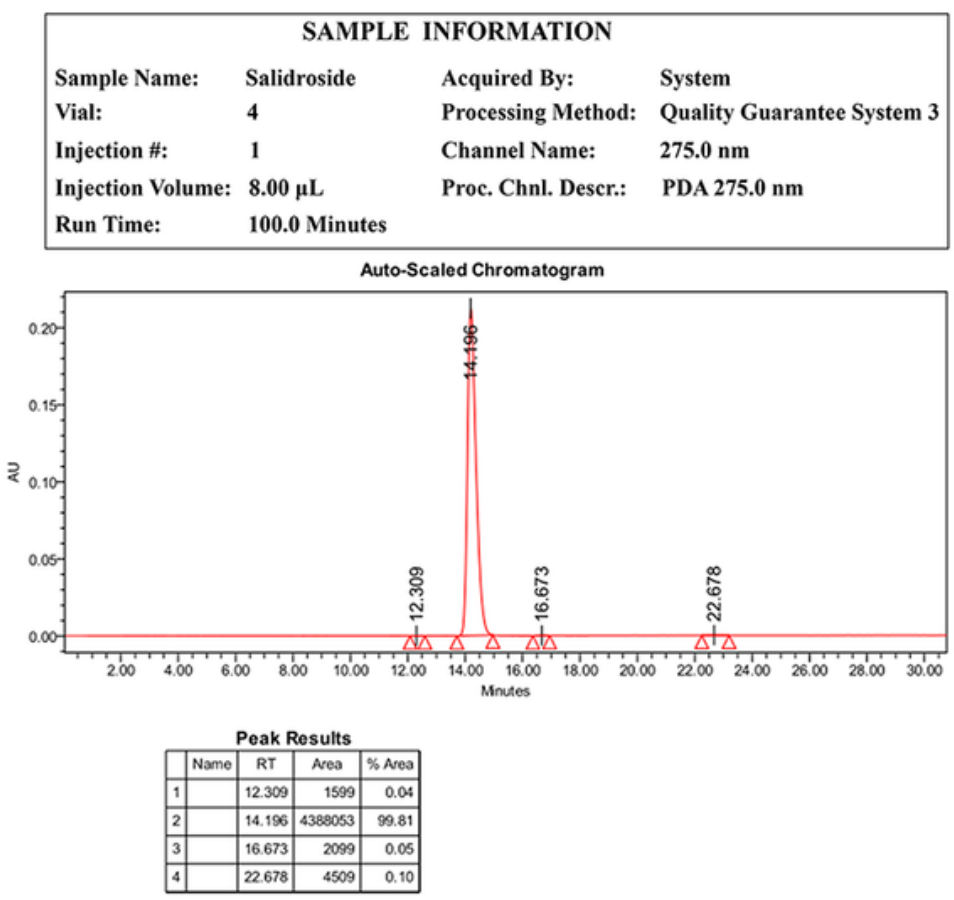

\section{C}

'иммм (рмsо) Nuclear Magnetic Resonance, NMR

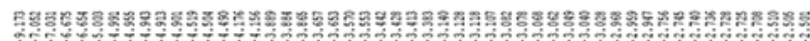

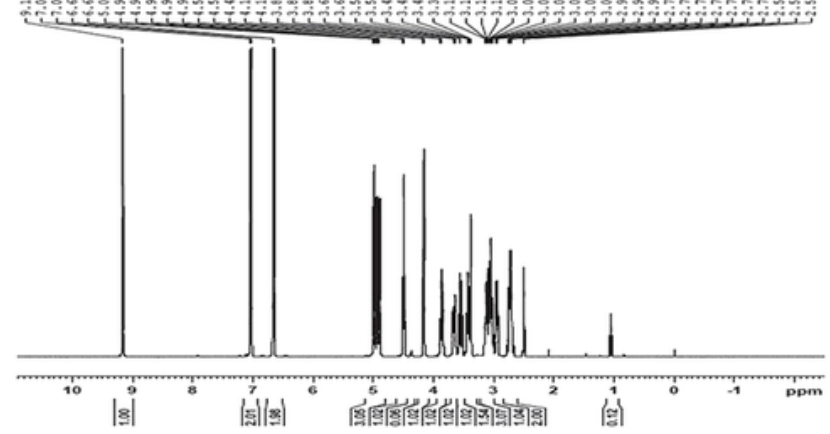

"C.NMR (DMSO)

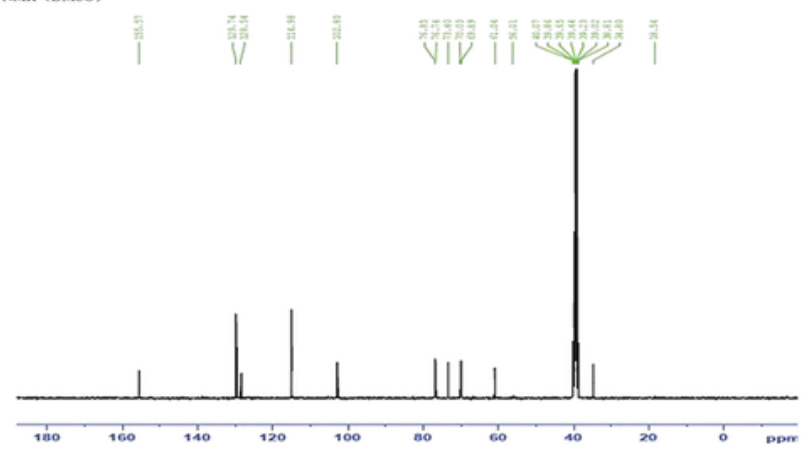

d

Mass Spectrometry, MS
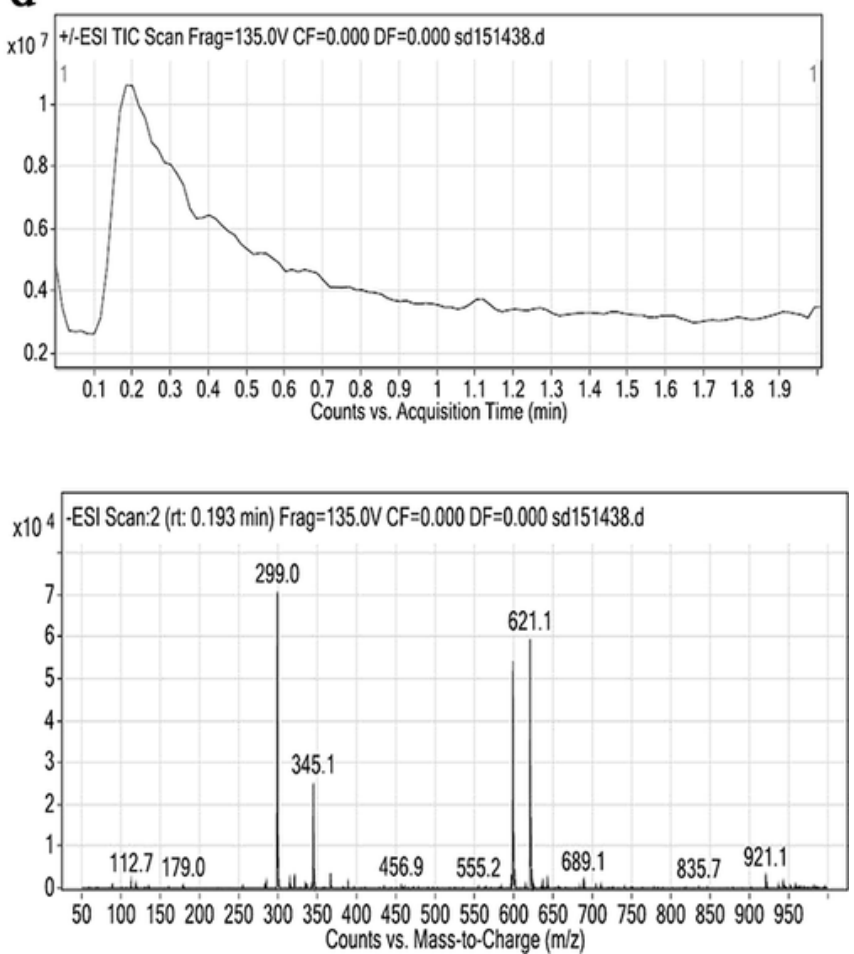

\section{Figure 1}

The purity and structure of SDS were detected using HPLC (b), NMR (c), and MS (d). 


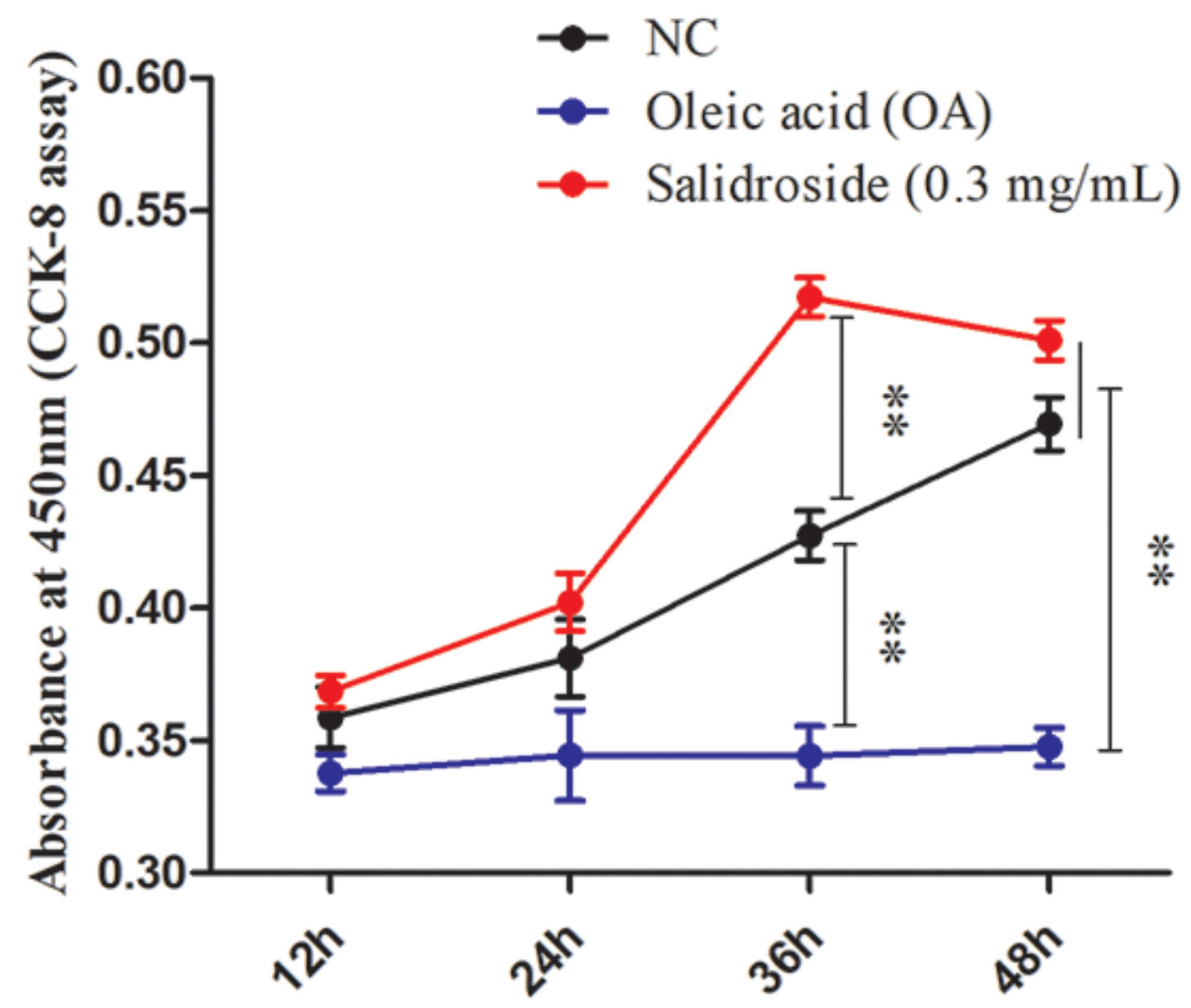

Figure 2

The OD values of hepatocytes treated with $0.3 \mathrm{mg} / \mathrm{mL}$ Salidroside for $12,24,36$, and $48 \mathrm{~h}$, respectively. Bars represent SEM; $* \mathrm{P}<0.05$ and $* * P<0.01$.

2

Figure 3

SDS inhibits OA-induced lipid accumulation in primary chicken hepatocytes. (a) The oil red 0 staining of chicken primary hepatocytes. (b) The mRNA expression abundances of lipid metabolism related genes.

(c) The protein level of PPARa was detected by western blot. Replications $=3$. Bars represent SEM; $* \mathrm{P}<$ 0.05 and ${ }^{*} \mathrm{P}<0.01$. 


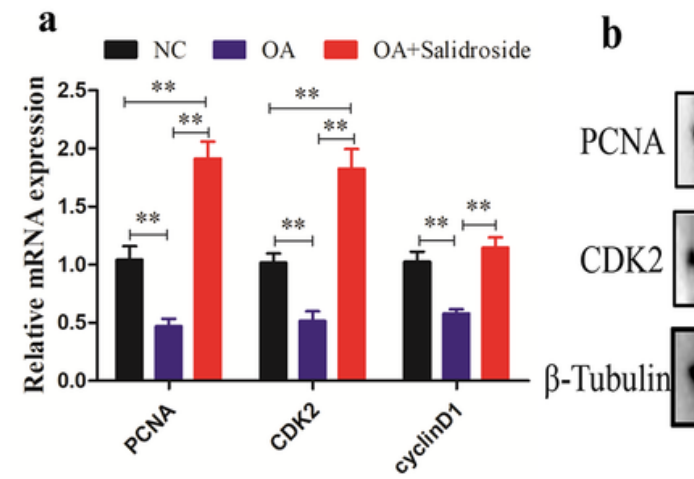

d

DAPI

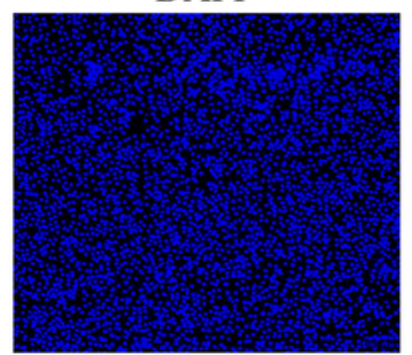

$\mathrm{NC}$

$\mathrm{OA}$
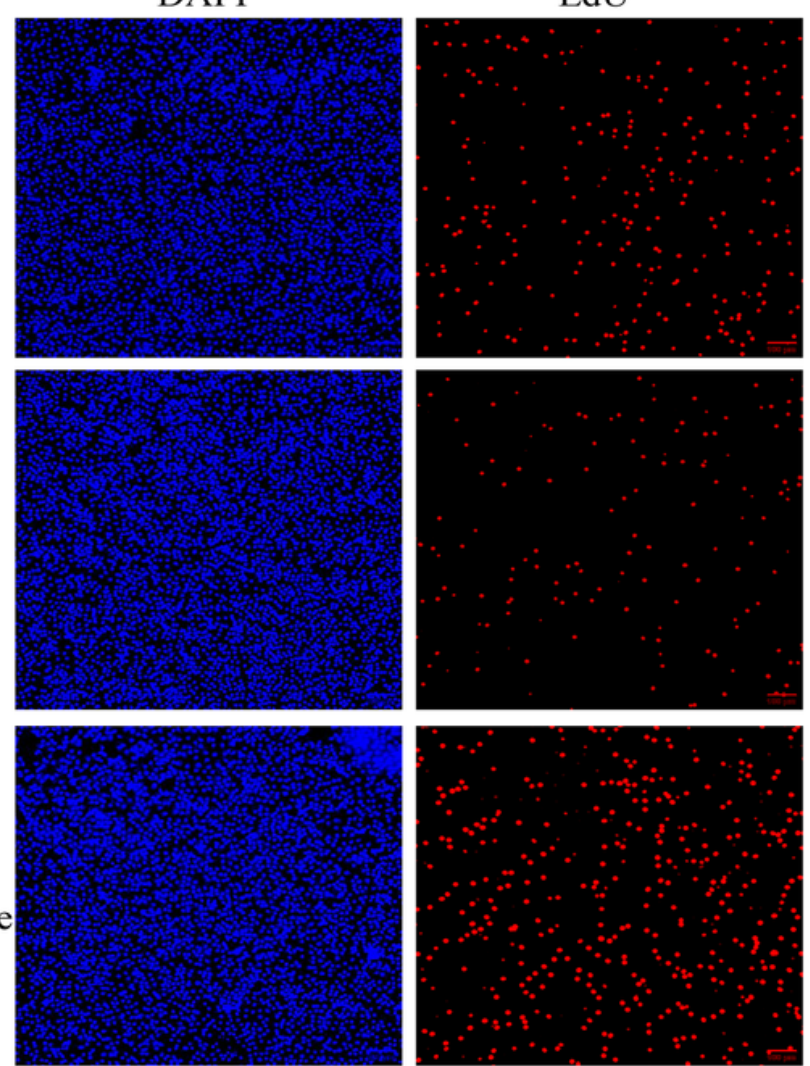

$\mathrm{OA}+$

Salidroside

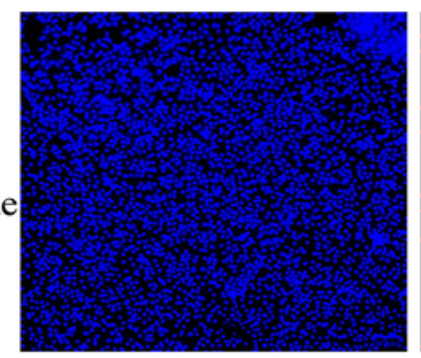

c

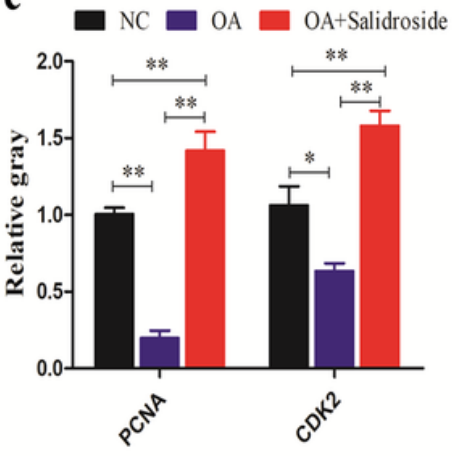

Overlay

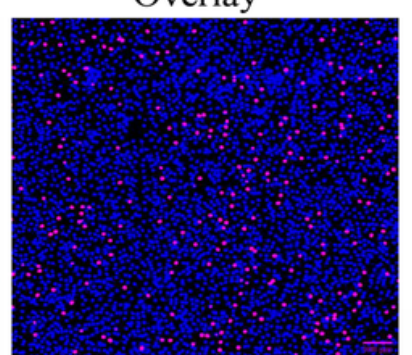

e ${ }_{\mathrm{OA}+\text { Salidroside }}^{\mathrm{OA}}$
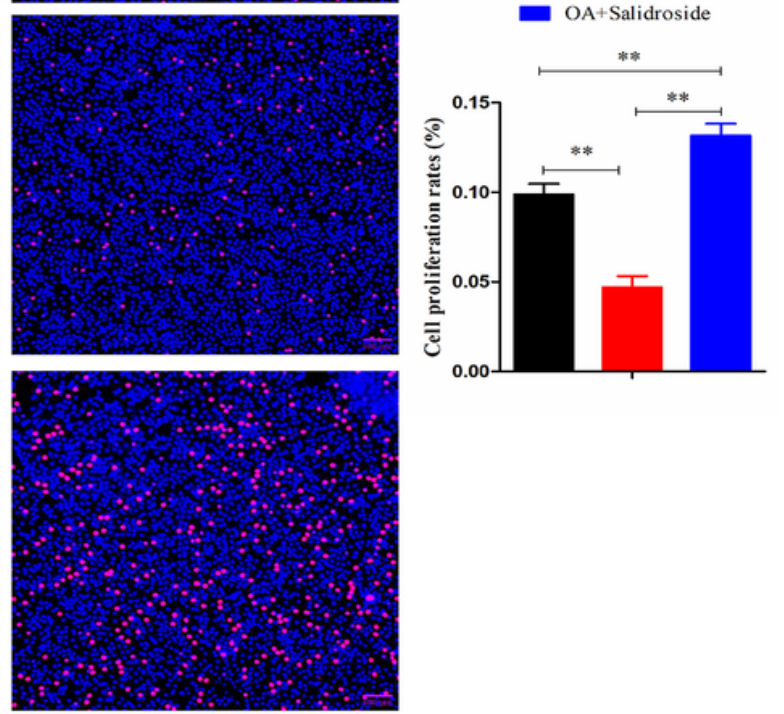

\section{Figure 4}

SDS promotes hepatocyte proliferation in OA-induced fatty liver model. (a) The mRNA expression of proliferation related genes. (b, c) The protein expression of PCNA and CDK2 were detected by western blot. (d, e) EdU staining-positive hepatocytes were detected by EdU kit. EdU (red), DAPI (blue); Replications $=3$. Bars represent SEM; ${ }^{*} \mathrm{P}<0.05$ and ${ }^{\star *} \mathrm{P}<0.01$. 

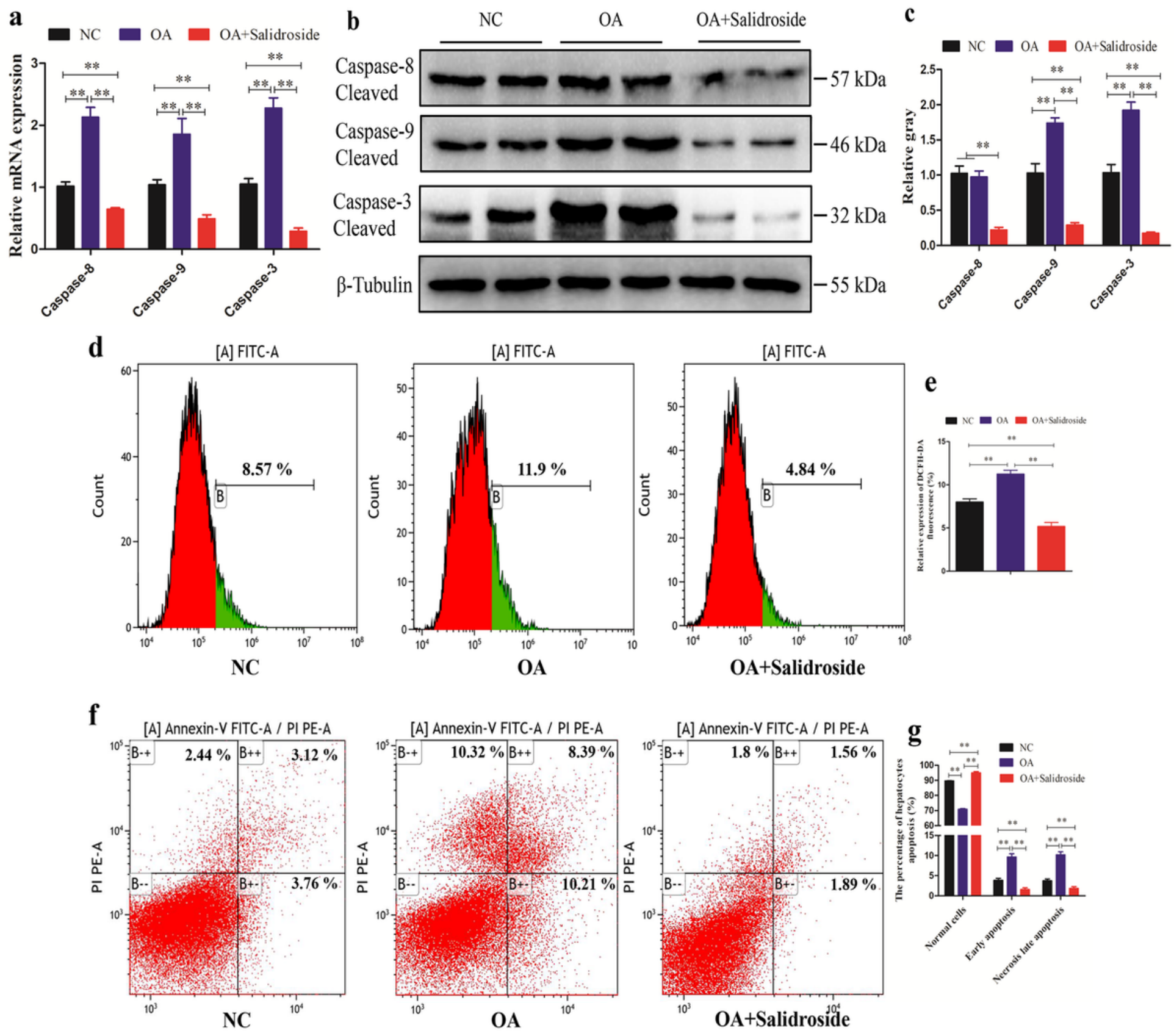

\section{Figure 5}

SDS inhibits hepatocyte apoptosis in an OA-induced fatty liver model. (a) The mRNA expression of apoptosis related genes (Caspase-8, Caspase-9, and Caspase-3). (b, c) Western blot analysis revealed the levels of Caspase-8, Caspase-9, and Caspase-3 cleavage. (d, e) ROS levels in chicken primary hepatocytes were determined by FITC-staining flow cytometry with an excitation wavelength of $488 \mathrm{~nm}$ and an emission wavelength of $525 \mathrm{~nm}$. ( $\mathrm{f}, \mathrm{g})$ Apoptotic hepatocytes were detected by annexin V-FITC/PIstaining flow cytometry. ROS: reactive oxygen species; NC: negative control. OA: oleic acid. Replications = 3. Bars represent SEM; *P $<0.05$ and ${ }^{\star *} \mathrm{P}<0.01$. 


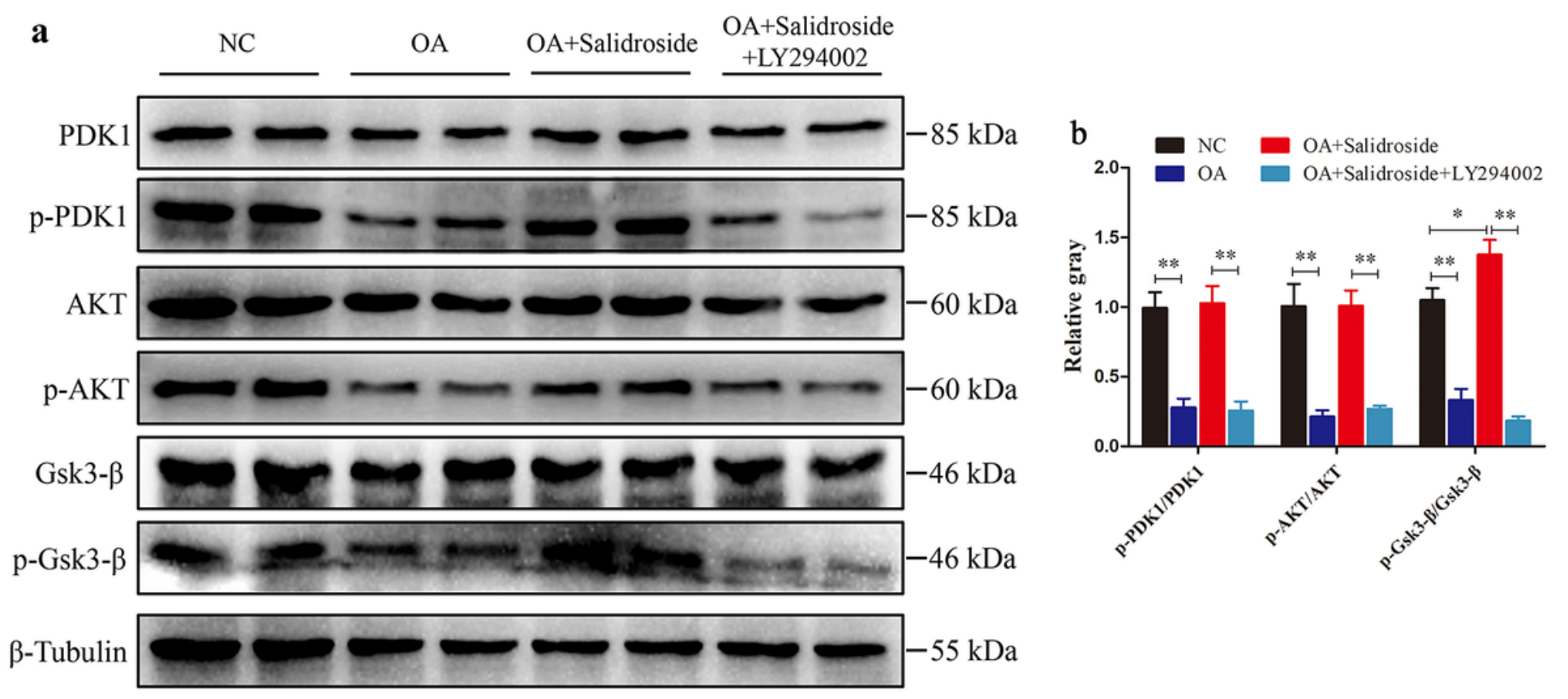

\section{Figure 6}

SDS targeted the PI3K/AKT/Gsk3- $\beta$ pathway. $(a, b)$ The total protein and phosphorylation levels of PDK1, AKT, and Gsk3- $\beta$ were detected by western blot. NC: negative control. OA: oleic acid. LY294002: PI3K inhibitor. Replications $=3$. Bars represent SEM; $* P<0.05$ and ${ }^{*} \mathrm{P}<0.01$.
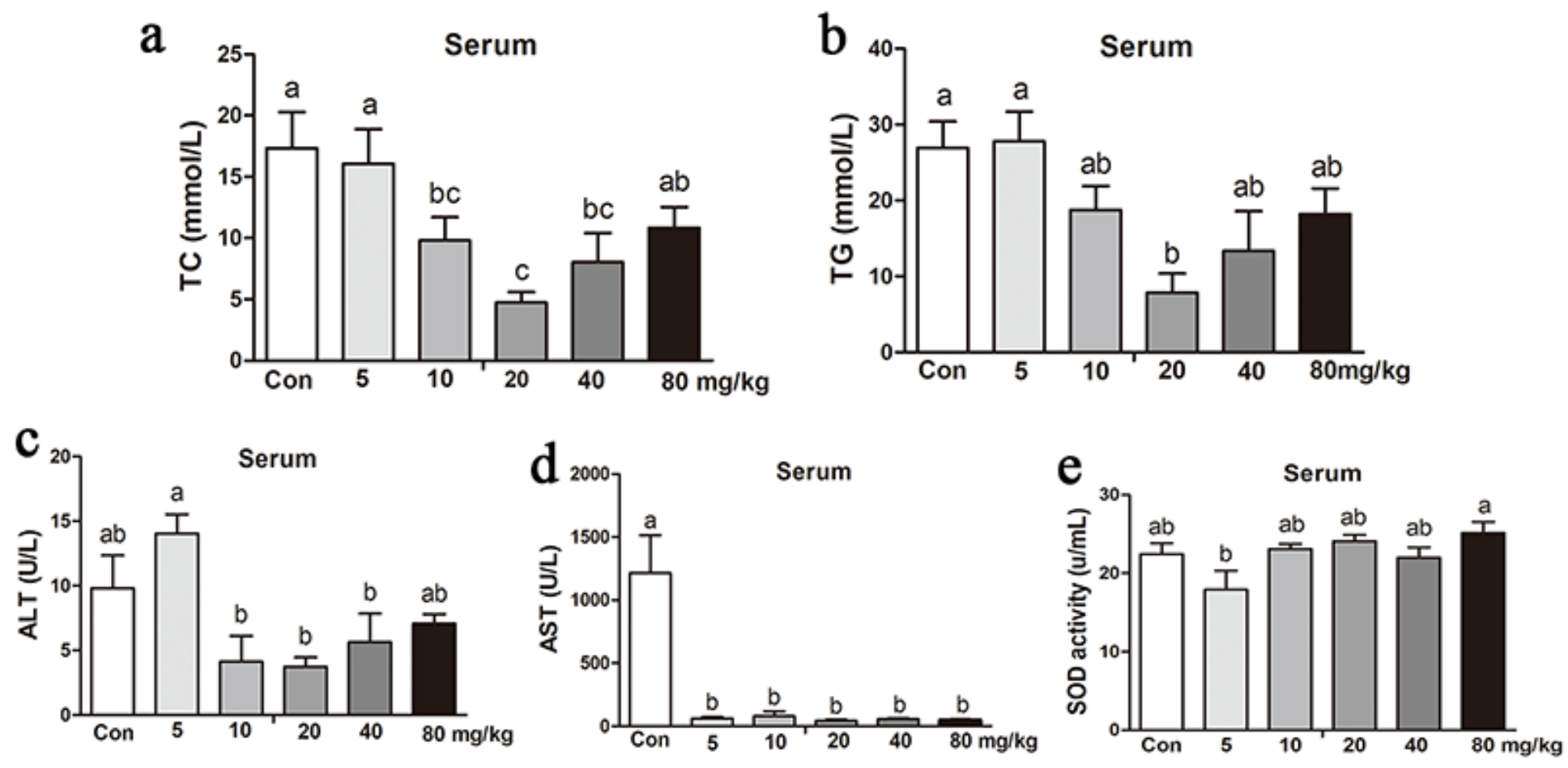

Figure 7

Serum biochemical parameters: (a) TC (total cholesterol), (b) TG (triglyceride), (c) ALT (alanine aminotransferase), (d) AST (aspartate aminotransferase), and (e) SOD (superoxide dismutase) were 
measured with ELISA kits. Bars represent SEM $(n=9)$. Different letters indicate significant differences $(P$ $<0.05)$.
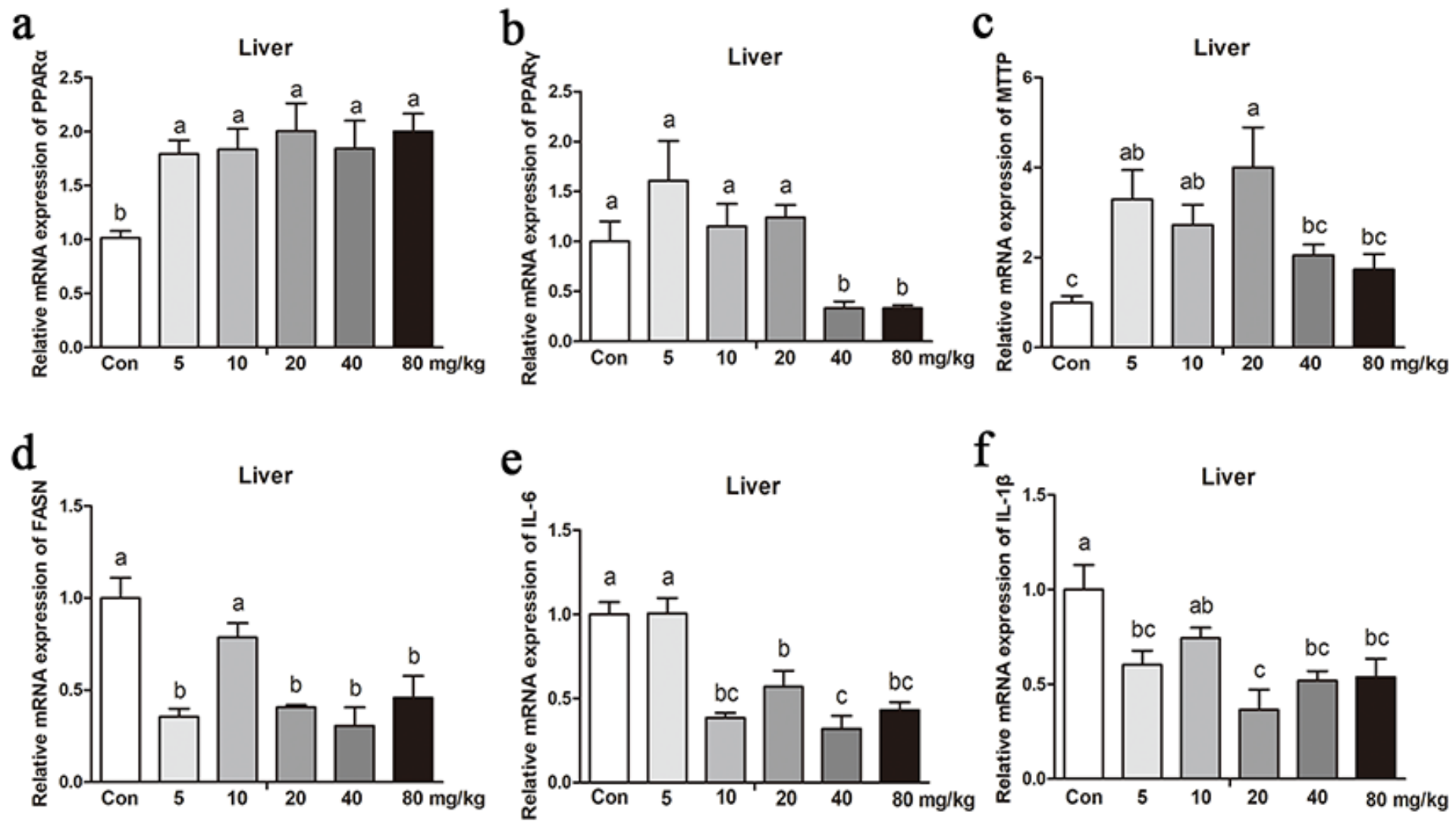

Figure 8

Feeding SDS affected gene expression. ( $a, b, c$, and d) The mRNA expression of lipid metabolism related genes in chicken liver. $(e, f)$ The mRNA expression level of pro-inflammatory cytokines (IL- 6 and IL-1 $\beta$ ) in chicken liver. Bars represent SEM $(n=3)$. Different letters indicate significant differences $(P<0.05)$. 

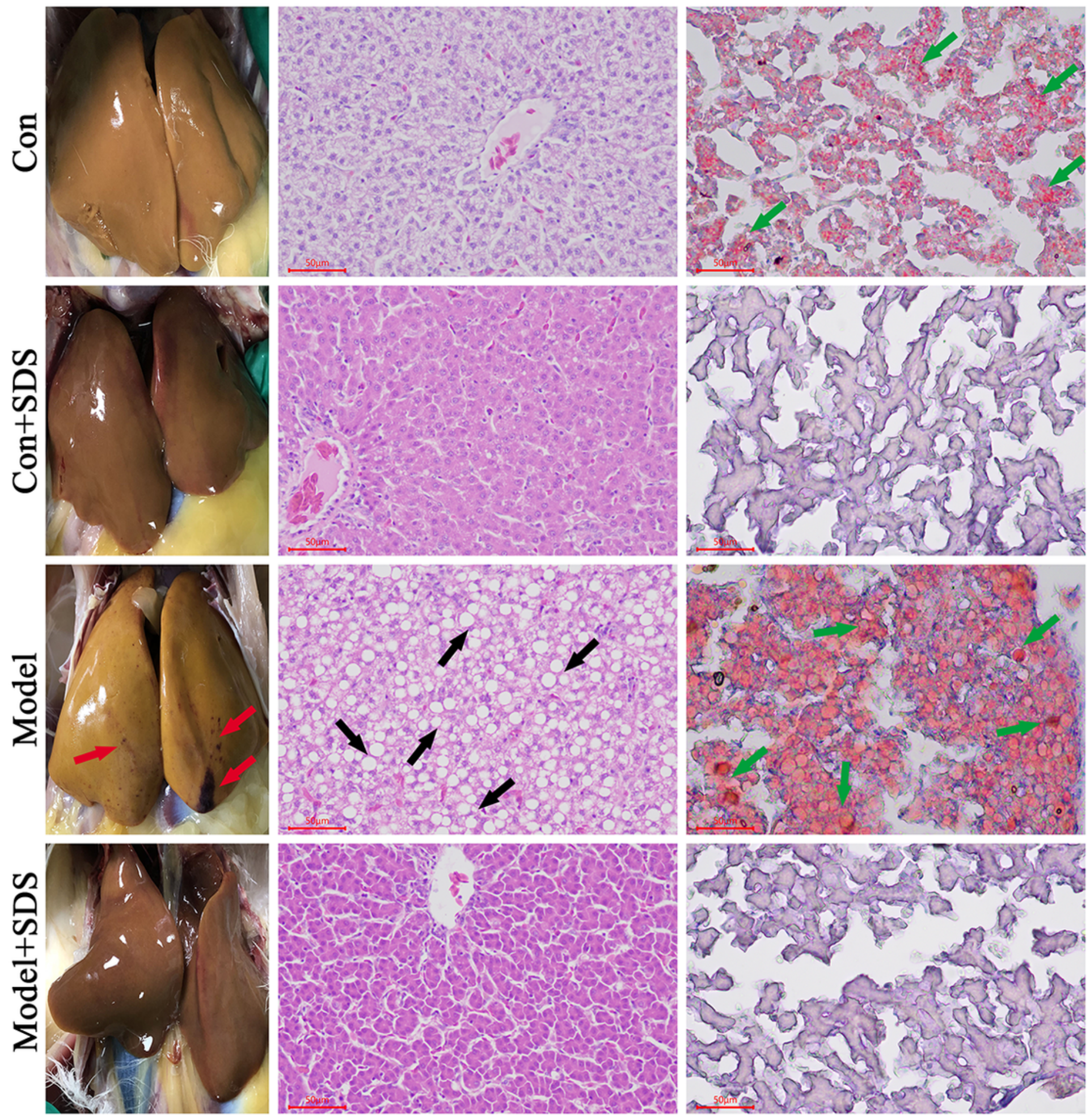

Figure 9

The morphological and histological characteristics of chicken liver. Hematoxylin-eosin (HE) staining and Oil red 0 staining were magnified $200 x$. The black arrow indicates the "fatty vacuoles", the green arrow indicates the "lipid droplets", and the red arrow indicates severe pathological changes of the fatty liverhemorrhagic syndrome of laying hens. 

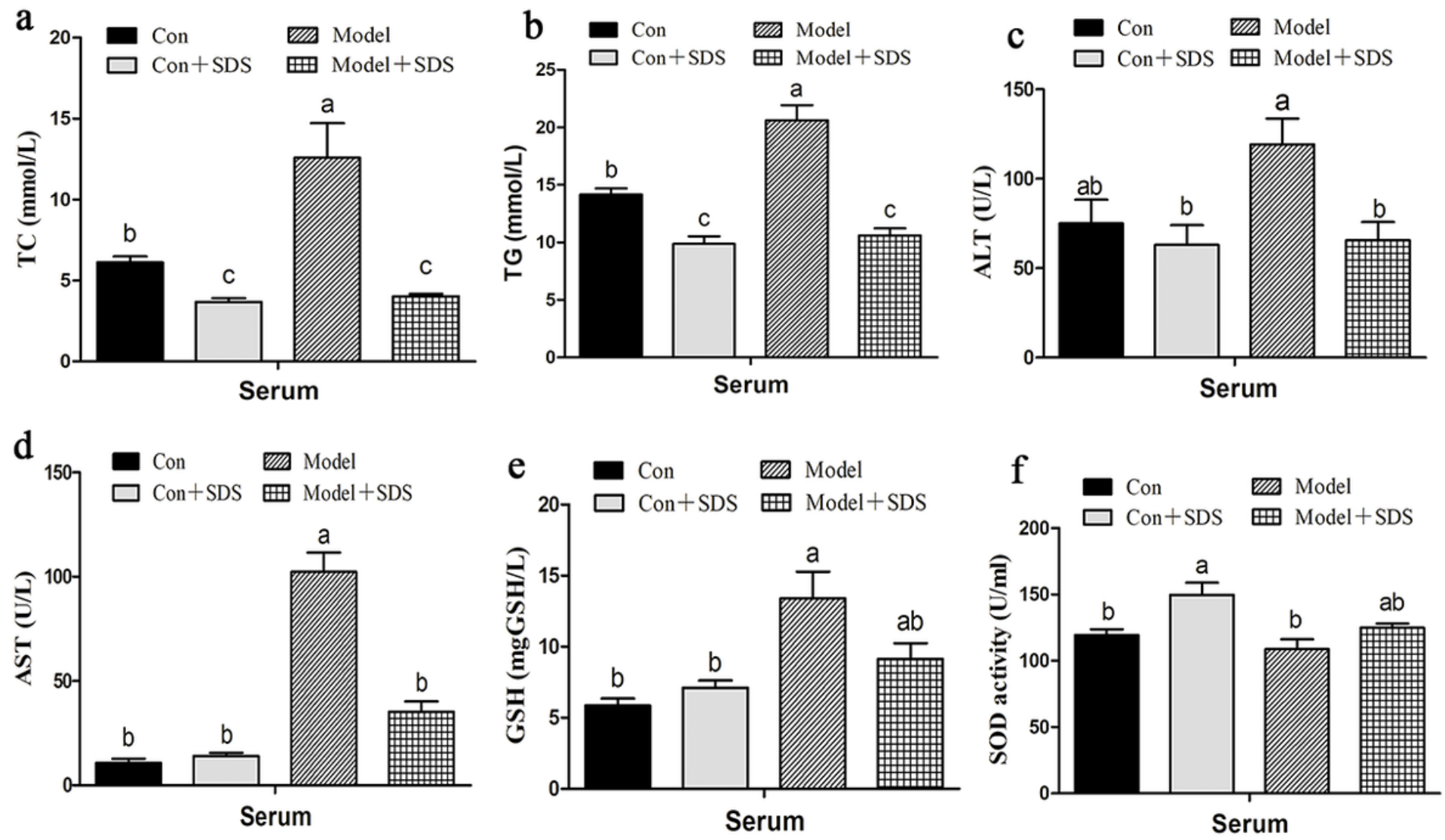

Figure 10

Serum biochemical parameters (a) TC (total cholesterol), (b) TG (triglyceride), (c) ALT (alanine aminotransferase), (d) AST (aspartate aminotransferase), (e) GSH (glutathione), and (f) SOD (superoxide dismutase) were detected in chicken blood. Bars represent SEM $(n=9)$. Columns without the same lowercase indicate significant differences between the groups $(P<0.05)$. 

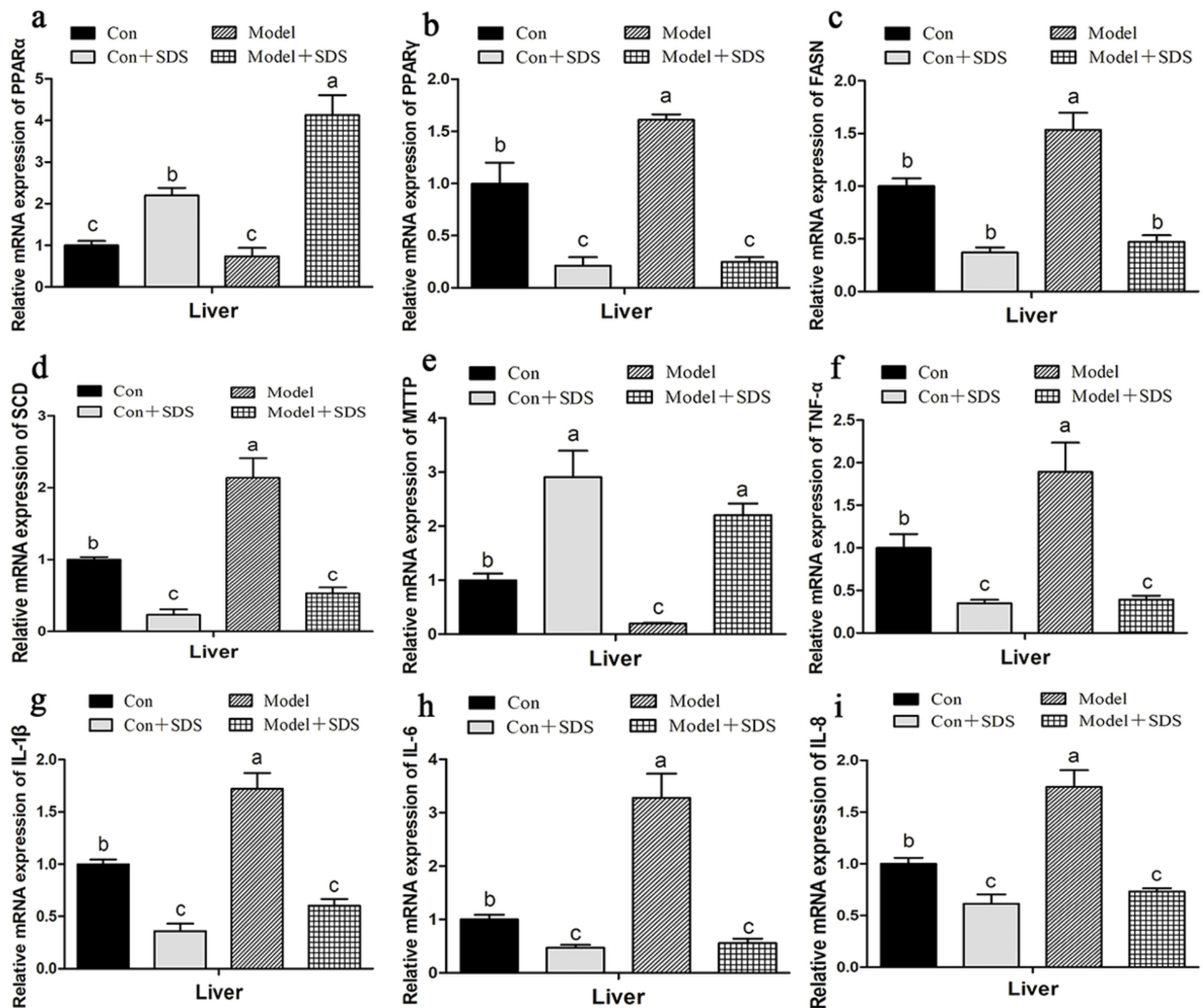

\section{Figure 11}

SDS alleviates high-fat diet-induced hepatic steatosis and inflammatory response of laying hens in vivo. ( $a, b, c, d$, and e) The mRNA expression of lipid metabolism related genes (PPARa, PPARy, FASN, SCD, and MTTP). ( $f, g, h$, and i) The mRNA expression level of pro-inflammatory cytokines (TNF-a, IL-1 $\beta$, IL-6, and IL-8) in chicken liver. Bars represent SEM $(n=3)$. Different lowercase indicates significant differences between the groups $(P<0.05)$.

\section{Supplementary Files}

This is a list of supplementary files associated with this preprint. Click to download. 
- SupplementalMaterial.docx

Page 28/28 